\title{
Research Paper \\ The Effect of an 8-week NASM Corrective Exercise Program on Upper Crossed Syndrome
}

\author{
*Mahsa Abdolahzadeh ${ }^{1} \odot$, Hassan Daneshmandi ${ }^{2} \odot$
}

1. Department of Sports Injuries and Corrective Exercise, Shafagh Institute of Higher Education, Tonekabon, Iran.

2. Department of Sports Injuries and Corrective Exercise, Faculty of Physical Education and Sport Sciences, University of Guilan, Rasht, Iran.

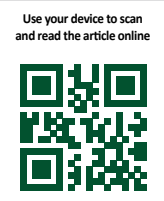

Citation: Abdolahzadeh M \& Daneshmandi H. [The Effect of an 8-week NASM Corrective Exercise Program on Upper Crossed Syndrome (Persian)]. Journal of Sport Biomechanics. 2019; 5(3):156-167. https://doi.org/10.32598/biomechanics.5.3.3

doi https://doi.org/10.32598/biomechanics.5.3.3

Corrective exercises, Upper crossed syndrome, NASM
Key words:

\section{ABSTRACT}

Article Info:

Received: 05 Feb 2019

Accepted: 16 Aug 2019

Available Online: 01 Dec 2019

\section{Extended Abstract}

\section{Introduction}

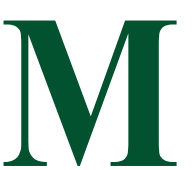

uscle imbalance can affect the body's natural alignment and cause a variety of postural abnormalities [1]. Improper posture and long-term work tasks can lead to musculoskeletal disorders [2]. Early and timely identification of these postural defects and their treatment can reduce its complications and help save time and money [3]. Muscle imbalance can have serious and known consequences in the body [1]. Upper Crossed Syndrome (UCS) occurs in the neck and shoulder girdle [4]. This syndrome is a type of musculoskeletal system involvement that results in shortening of the upper posterior and anterior muscles in the neck, which are tonic muscles (e.g. pectoralis major muscle, upper trapezius, levator scapula, sternocleidomastoid) and the anterior deep muscles of the neck and posterior shoulder girdle, which are mainly phasic (e.g. Rhomboid major, middle and lower trapezius muscles, serratus anterior muscle, and deep neck flexors) are inhibited and weakened. Postural changes seen in UCS include forward head, rounded shoulders, and thoracic kyphosis [5].

\section{* Corresponding Author:}

Mahsa Abdolahzadeh, MA.

Address: Department of Sports Injuries and Corrective Exercise, Shafagh Institute of Higher Education, Tonekabon, Iran.

Tel: +98 (911) 8837761

E-mail: mahsa.abdolahzadeh@gmail.com 
There have been several reports of osteoarthritis of the temporomandibular joint due to forward head and mechanical pain in the head [4]. There are also some reports of radicular pain in the arms and hands due to osteoarthritis of the neck due to UCS [2]. Such adverse secondary changes resulting from this syndrome are also present in people with thoracic kyphosis in the glenohumeral joint $[4,11]$.

\section{Methods}

In this study, 30 female students [15] with forward head posture $>46$ degrees [16], forward shoulder posture $>52$ degrees [16] and thoracic kyphosis $>42$ degrees [17] were selected as samples using purposive sampling method and randomly divided into groups of control and intervention. Participants in the intervention group received 8 weeks of corrective exercise, 3 sessions per week, each for 30$70 \mathrm{~min}$. National Academy of Sports Medicine (NASM) principles were used to develop the training program. The program follows certain training protocols in designing and implementing corrective exercises. It consists of four stages of inhibition, stretch, activation and coherence [9].

The head-forward and shoulder-forward angles were measured using side photography [19], the kyphosis angle was measured using a flexible ruler $(r=0.093)$ [20] before and after intervention. The type of movements was determined by referring to specialists and resources of movement therapy and then finalized and implemented through a pilot study on some study samples [21]. The Shapiro-Wilk test was used to measure the normal distribution of data. In order to analyze the data obtained from pre-test and posttest phases, pair $t$ test was used, and ANOVA test was used to compare the study changes.

\section{Results}

Table 1 presents the characteristics of participants and test results. Due to the long and incorrect sitting position and repetitive use of the upper limbs in students, there is a possibility that the balance of the muscles of the upper extremity is disturbed. Since muscle imbalances in the upper quarter of the body increase the risk of UCS, and UCS is associated with three postures of head forward, rounded shoulder and thoracic kyphosis, the exercises in this study were comprehensively and simultaneously based on these three abnormalities. The people with UCS need to pay special attention to the issue of muscle balance while sitting, in addition to correcting the posture of the head, neck and back. The results showed the positive impact of exercise based on NASM principles on muscle balance and correcting head forward, rounded shoulder and thoracic kyphosis postures.

\section{Discussion}

UCS is commonly seen in people who sit for long periods of time or in people who apply frequent overload patterns to upper limbs [8,9]. Corrective exercises have been reported to be one of the most effective ways to restore performance [23]. Eight weeks of corrective exercises regulates muscle activity and musculoskeletal disorders in the upper body [24]. In this study, the four-step NASM-based corrective protocol was focused on all three abnormalities caused by UCS at the same time, and is consistent with the Janda approach and the Bruegger's exercise [8]. Researchers have shown that strength training affects the length of the muscle tendon, displacing different parts of the skeleton and stabilizing the ligaments. On the other hand stretching exercises act as coordinator of agonist and antagonist muscles. Thus, such exercises increase the length of the muscles on the concave side, the muscle power and strength on the convex side, and thus reduce the rate of postural abnormalities [37]. We attempted to apply the exercise program more in a closed chain of motion and more in a weight-bearing position to simulate real-life activities [36].

\section{Conclusion}

In general, it seems that the use of corrective exercises can lead to improvements in flexibility and strength following

Table 1. Characteristics of participants before and after intervention (Mean \pm SD)

\begin{tabular}{cccccccccc}
\hline \multirow{2}{*}{ Characteristics } & \multicolumn{4}{c}{ Control Group ( $\mathbf{n}=15)$} & \multicolumn{5}{c}{ Intervention Group (n=15) } \\
\cline { 2 - 9 } & Pre-test & Post-test & T & P & Pre-test & Post-test & T & P \\
\hline Head forward & $49.5 \pm 3.01$ & $49.6 \pm 3.07$ & -1.23 & 0.23 & $50.0 \pm 1.38$ & $44.7 \pm 1.69$ & 13.36 & $* 0.001$ \\
Rounded shoulder & $55.0 \pm 1.50$ & $55.1 \pm 1.40$ & -0.37 & 0.71 & $55.0 \pm 1.06$ & $50.5 \pm 0.90$ & 20.32 & $* 0.001$ \\
\hline Thoracic kyphosis & $47.0 \pm 2.06$ & $47.1 \pm 2.04$ & -1.24 & 0.23 & $48.2 \pm 1.46$ & $42.36 \pm 1.45$ & 29.78 & $* 0.001$ \\
\hline
\end{tabular}


the correction of postural abnormalities. Hence, the use of corrective exercises to reduce UCS abnormalities and improve strength and flexibility is recommended.

\section{Ethical Considerations}

Compliance with ethical guidelines

The present study ethically approved by the Research Ethics Committee of Sport Sciences Research Institute code:.1398.628 IR.SSRI.REC.

Funding

This study was extracted from the master thesis of first author approved by Department of Sport Injuries and corrective exercises, Shafagh Institute of Higher Education, Tonekabon, Iran.

Authors' contributions

Conceptualization, methodology, validation, analysis, research and supervision: All authors; writing original draft and resources: Mahsa Abdolahzadeh. Editing \& review: Hassan Daneshmandi.

Conflicts of interest

The author declared no conflict of interest 


\title{
تأثير هشت هفته تمرينات اصلاحى مبتنى بر اصول NASM بر سندرم متقاطع فوقانى
}

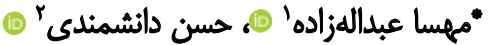 \\ 1. كروه آسيبشناسى ورزشى و حركات اصلاحي، مؤسسه آموزش عالى شفق تنكابن، مازندران، ايران.

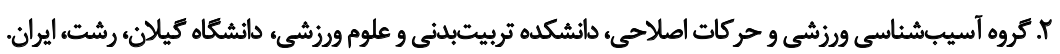

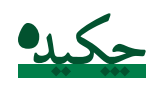

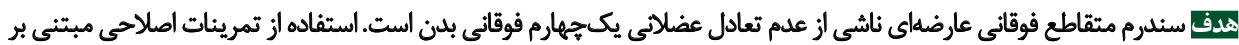

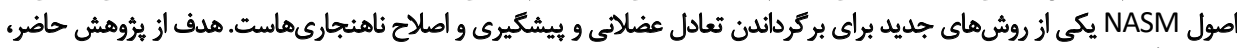

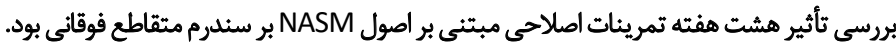

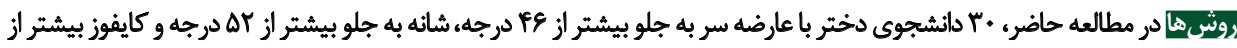

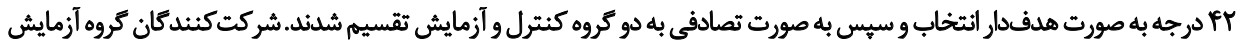

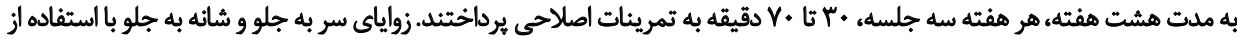

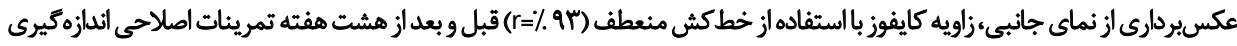

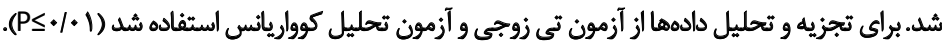

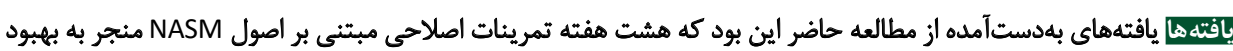

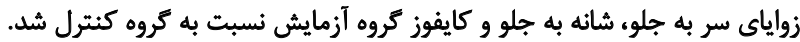

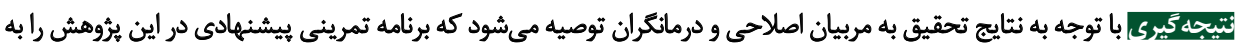
طور منظم اجراكنيد.

اطلاعات مقاله:

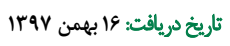

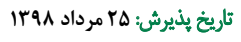

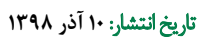

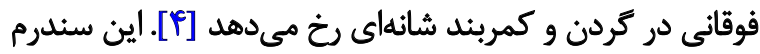

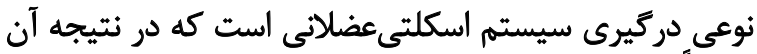

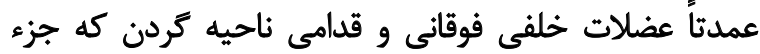

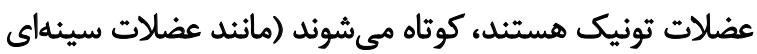

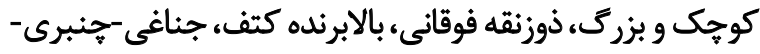

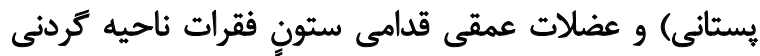

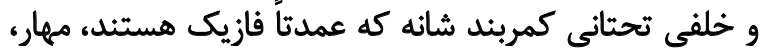

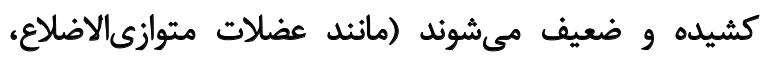

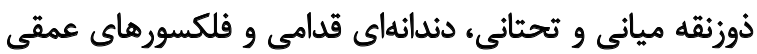

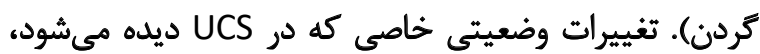

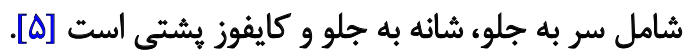

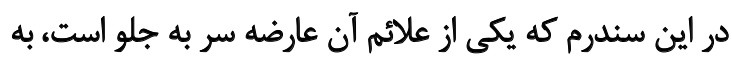

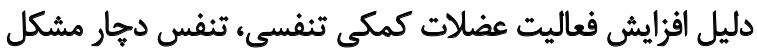

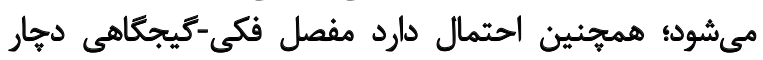

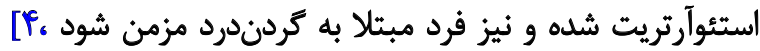

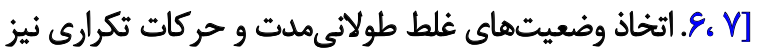

عدم تعادل عضلانى' ممكن است بر راستاى طبيعى بدن

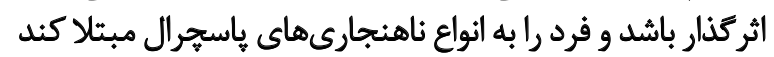

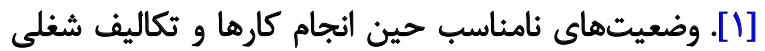

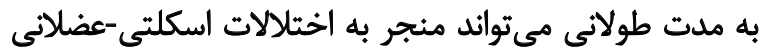

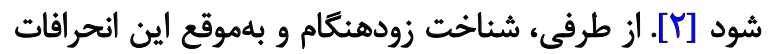

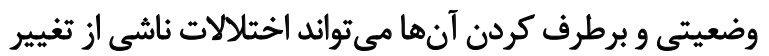

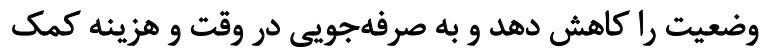

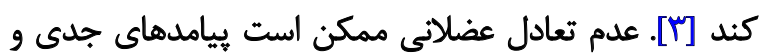

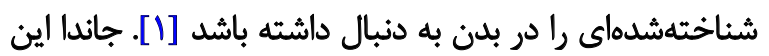

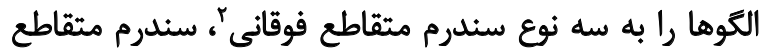

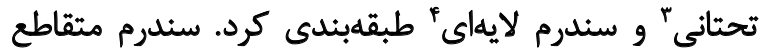

1. Muscle imbalance

2. Upper crossed syndrome (UCS)

3. Lower crossed syndrome (LCS)

4. Layer syndrome

نشائى: مازندران، تنكابن، مؤسسه آموزش عالى شفق، كروه آسيبشناسى ورزشى و حركات اصلاحى.

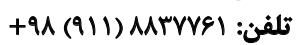
mahsa.abdolahzadeh@gmail.com : يست الكترونيكي: 
در اين ميان آكادمى ملى طب ورزش آمريكا (NASM) زنجيره

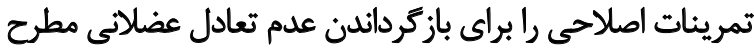

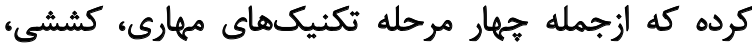

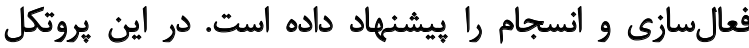

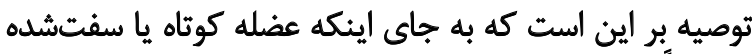

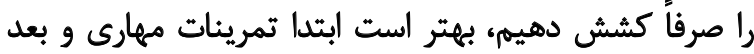

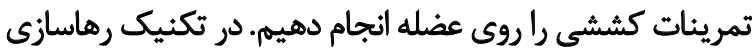

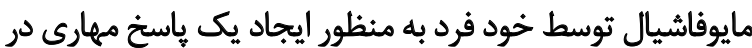

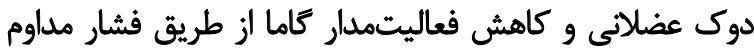

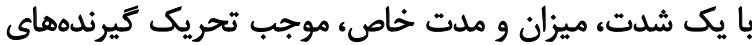

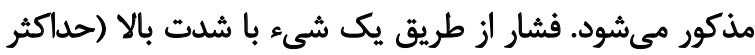

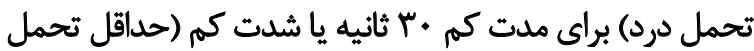

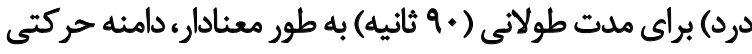

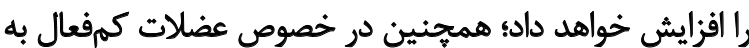

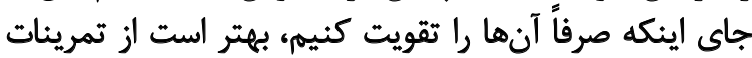

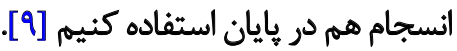

برايناساس، محققان با روشهاى كوناكون با تكيه بر اصول

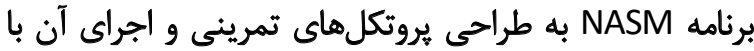

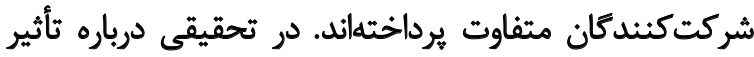

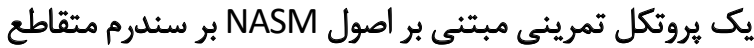

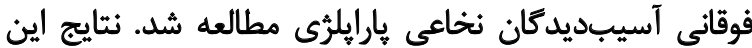

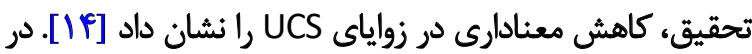

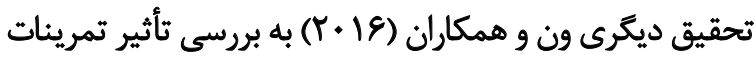

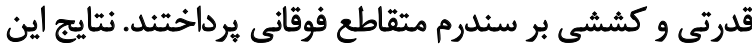

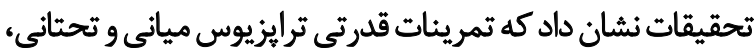

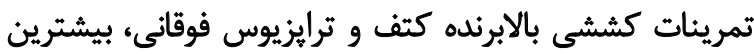

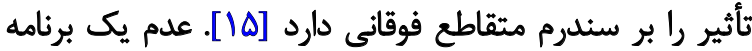

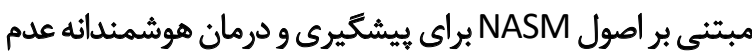

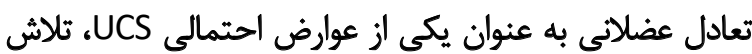

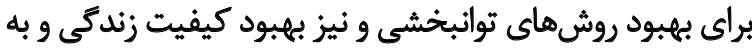

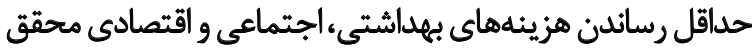

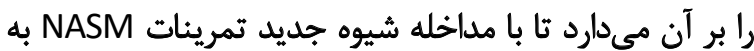

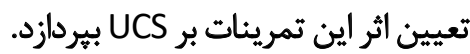

$$
\text { روش }
$$

تحقيق حاضر با توجه به اعمال مداخله، داشتن گروه كنترل

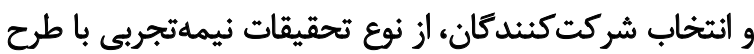

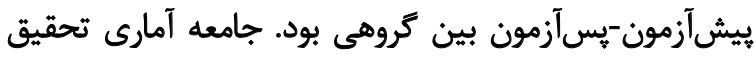
حاضر را دانشجويان غيرورزشكار دختر

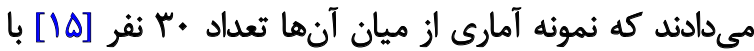

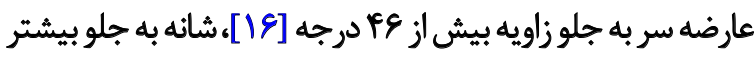

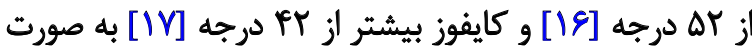

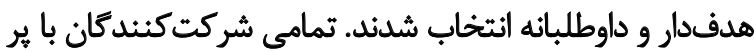
كردن فرم رضايت نامه در تحقيق شركت كردند. ابتدا اطلاعات
موجب تغييرات هاتولوزيكى در ديكر ساختارهاي اسكلتى-عضلاتى

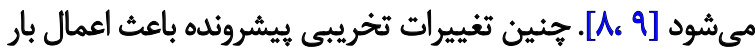

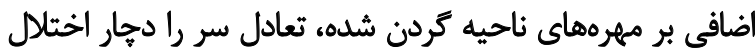

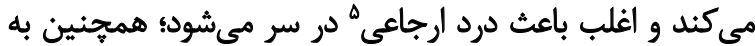

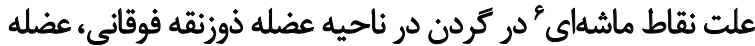

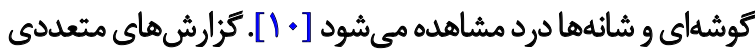

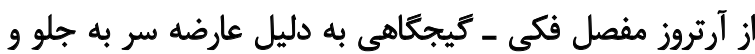

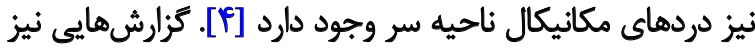

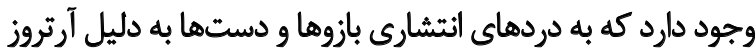

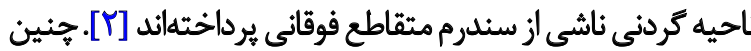

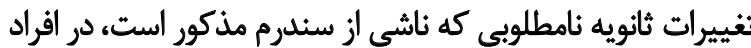

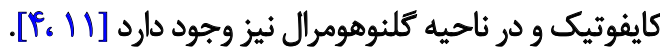

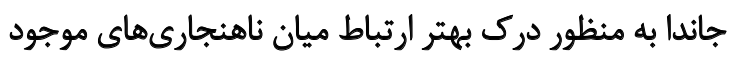

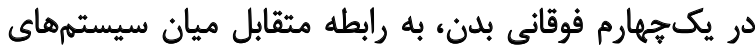

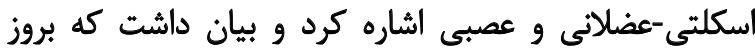

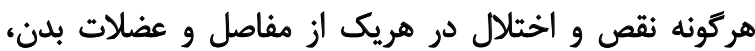

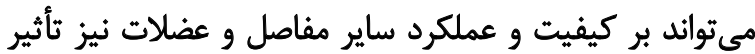

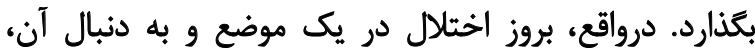

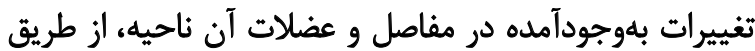

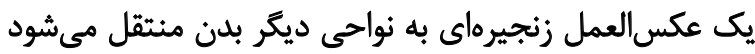

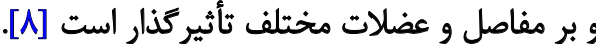

در كذشته روشهاي كوناتونى براي اصلاح اين سندرم وجود

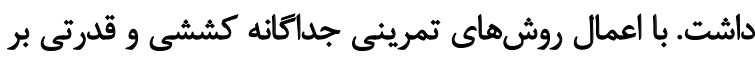

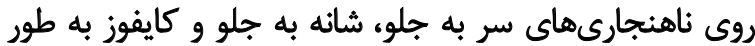

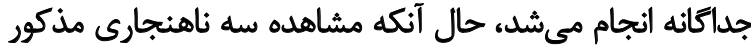

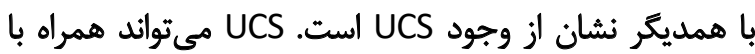

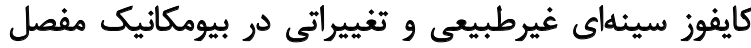

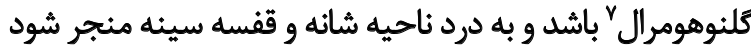

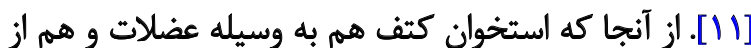

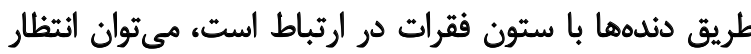

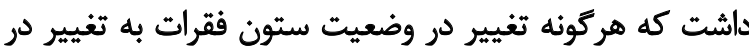

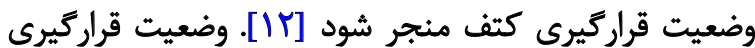

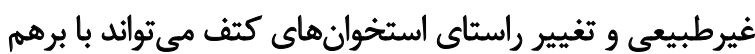

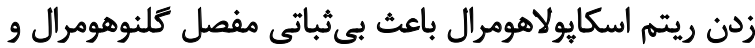

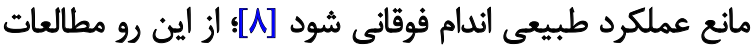

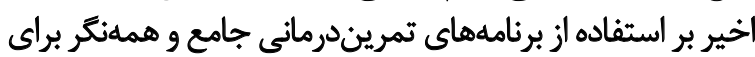

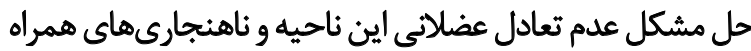

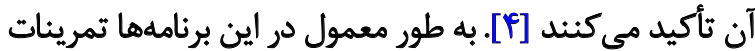

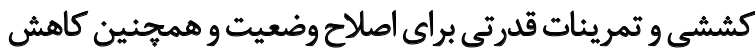

درد ناشى از اصلاح وضعيت در نظر كرفته شده است [1/]

5. Referral Pain

6. Trigger Points

7. Glenohumeral Joint 


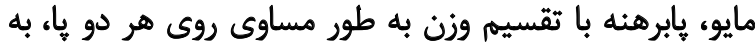

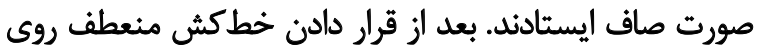

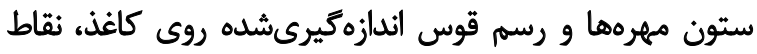

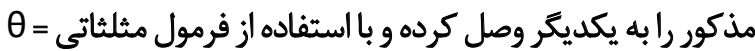

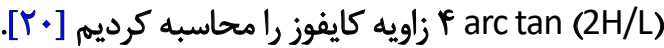

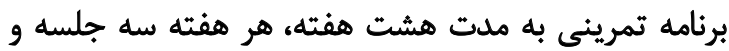

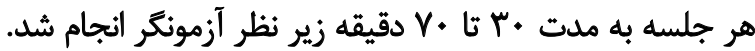

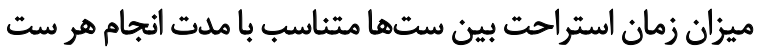

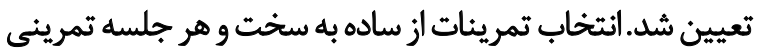

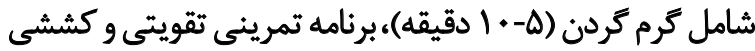

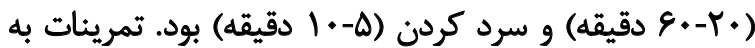

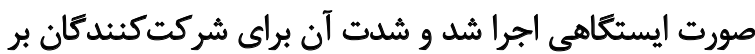

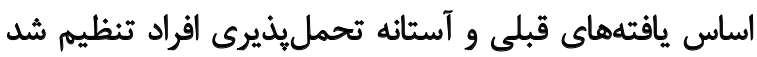

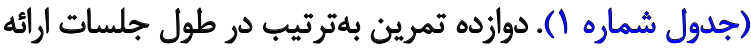

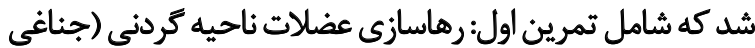

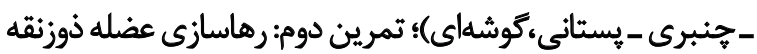

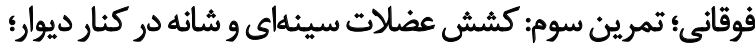

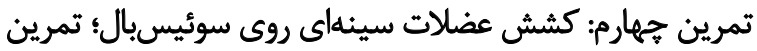

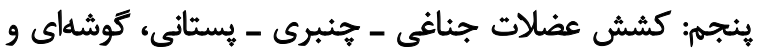

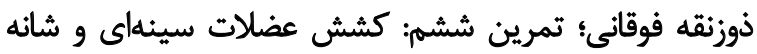

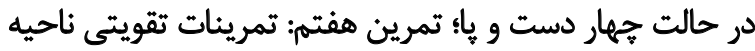

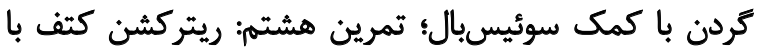

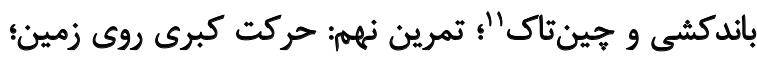

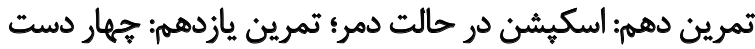

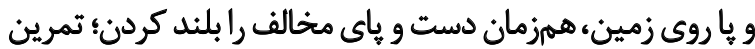

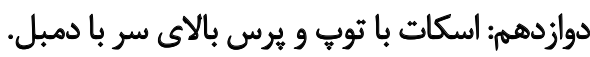

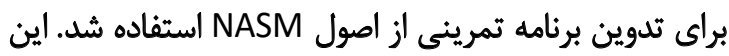

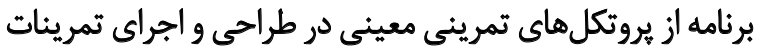

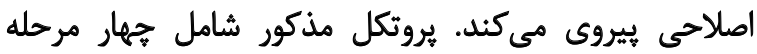

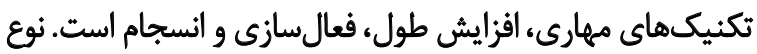

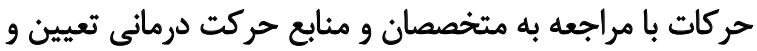

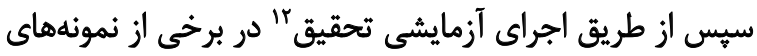

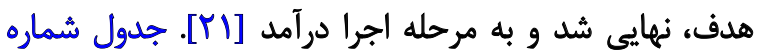

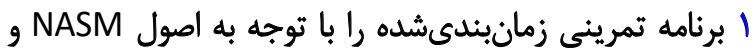

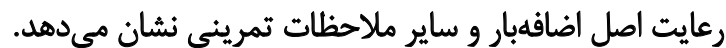

نثايج

اطلاعات مربوط به مشخصات دموكرافى نمونهها در جدول

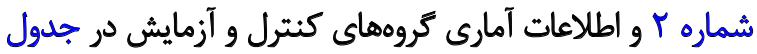

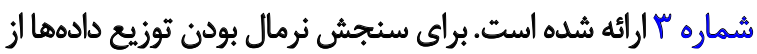

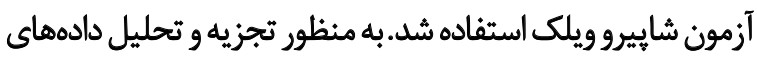

11. Chin Tuck

12. Pilot Study
دموكرافيك افراد شامل سن، قد، وزن و شاخص توده بدنى آنها

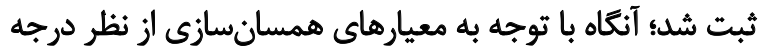

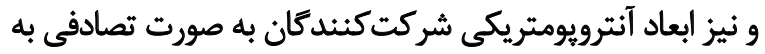

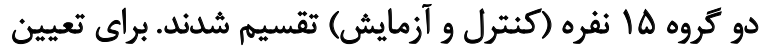

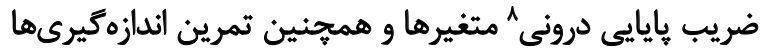

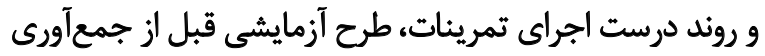

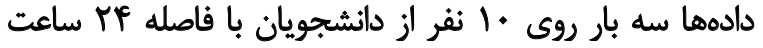

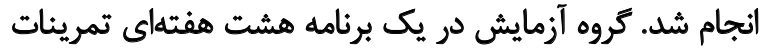

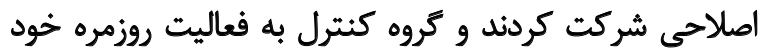

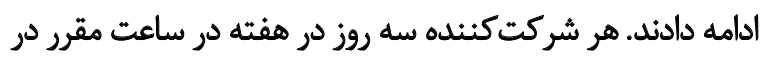

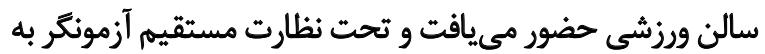

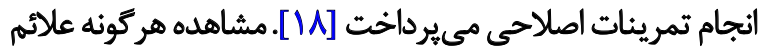

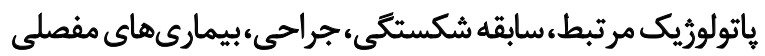

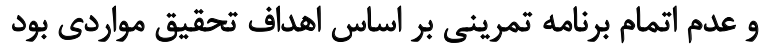

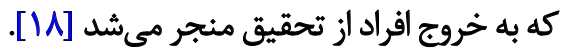

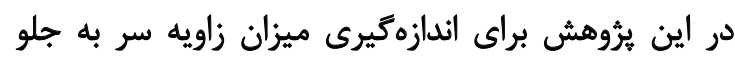

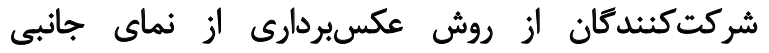

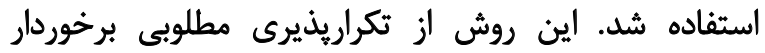

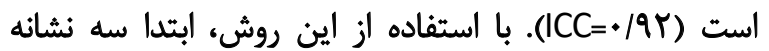

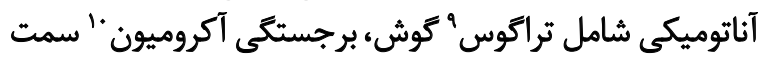

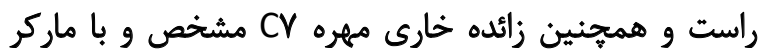

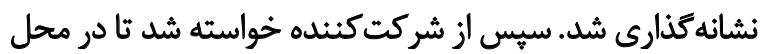

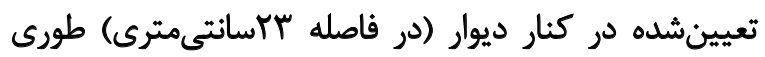

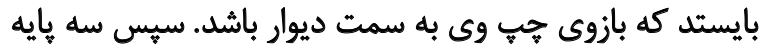

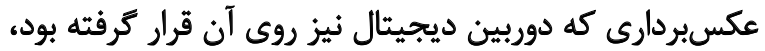

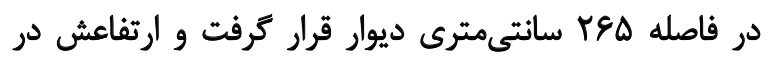

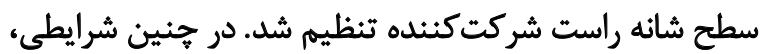

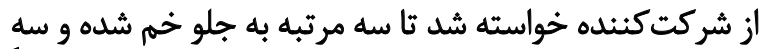

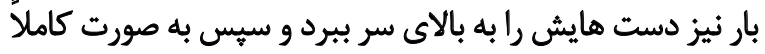

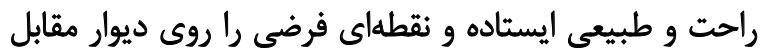

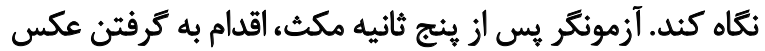

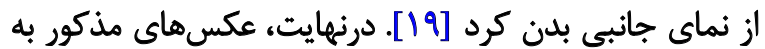

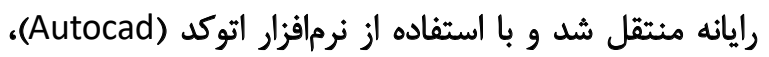

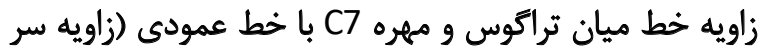

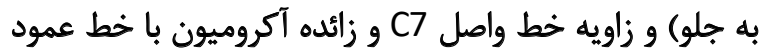

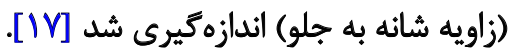
براى اندازهيرى انحناى يشتى ستون فقرات از خطكش

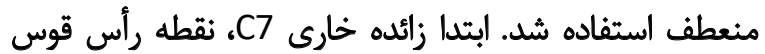

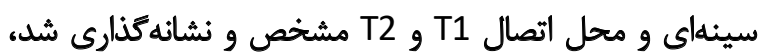

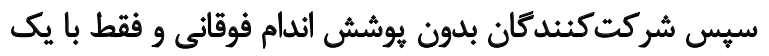

8.Interaclass Correlation Coefficient 9.Tragus

10. Acromion Process 
جدول ا. يُيشرفت برنامه تمرينات اصلاحي در جلسات تمرين

\begin{tabular}{|c|c|c|c|c|c|c|c|c|}
\hline هشتم & هفتم هفم & ششم & ينجم & جهارم & سوم & 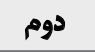 & 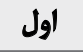 & هفته / شماره تمرين \\
\hline$r \times \Lambda$ & $r \times \wedge$ & $r \times 1$ & $r \times \Lambda$ & $r \times 1$ & $r \times 1$ & $r \times \Lambda$ & $r \times 1$ & جلسه اول \\
\hline$r \times \Lambda$ & $r \times A$ & $r \times 1$ & $r \times \Lambda$ & $r \times 1$ & $r \times 1$ & $r \times \Lambda$ & $r \times 1$ & جلسه دوم \\
\hline$r \times 1$ & $r \times A$ & $r \times 1$ & $r \times A$ & $r \times 1$ & $r \times A$ & $r \times 1$ & $r \times A$ & جلسه سوم \\
\hline$r \times 1$. & $r \times 1$. & $|x|$. & $r \times 1$. & $r|x|$ & $|r|$. & $r \times \mid$. & $|x|$. & جلسه اول \\
\hline$r \times 1$. & $r \times 1$. & $r \times 1$. & $r \times 1$. & $r \times 1$. & $r \times 1$. & $r \times 1$. & $r|x|$. & جلسه دوم \\
\hline$r \times u^{\prime}$ & $r \times l^{2}$ & $|x|$. & $|r|$ & $r \times 1$. & $r \times u^{2}$ & $r \times 1$. & $r|x|$. & جلسه سوم \\
\hline نهم & هشتم & هفتم هنم & ششمي & ينجم & جهارم & سوم & 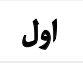 & هفته / شماره تمرين \\
\hline$r \times 1$ & $r \times 1$. & $|r| *$ & $r \times 1$. & $r \times 1$. & $r \times l^{2}$ & $r \times 1$. & $r|x|+$ & جلسه اول \\
\hline$r \times 1$ & $r \times 1$. & $|x|$ & $|r|$. & $r \times 1$. & $r \times 1$. & $r \times 1$. & $|x|$. & جلسه دوم \\
\hline$r \times 1$ & $r \times l^{2}$ & $|x|$. & $|r|$ & $r \times 1$. & $|x|$. & $|x|$. & $|x|$. & جلسه سوم \\
\hline$r \times u^{\prime}$ & relr & $r \times \mid r$ & rxir & rXIY & rxir & reir & 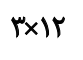 & جلسه اول \\
\hline$r \times 1$. & $r \times I r$ & $r \times \mid r$ & rxir & reir & reir & rxir & $r \times i r$ & جلسه دوم \\
\hline$r \times 1$. & relr & $r \times \mid r$ & rxir & reir & reir & rXIr & $r x i r$ & جلسه سوم \\
\hline هم⿻ & نهم⿻ & هشتم & هفتم & ششم & هنجم & جهارم & سوم & هفته / شماره تمرين \\
\hline $1 \times 8$ & $|x|$. & $|x|$. & $|x|$ & $|x|$. & $|x|$ & $|x|$. & $|x|$. & جلسه اول \\
\hline $1 \times 8$ & $f \times 1$. & $|x|$. & $|x|$ & $|x|$ & $|x|$. & $|x|$. & $|x|$. & جلسه دوم \\
\hline $1 \times 8$ & $f \times l$. & $|x|$. & $|x|$ & $|x|$. & $|x|$. & $f \times 1$. & $|x|$. & جلسه سوم \\
\hline$P \times 1$ & $P \times l$. & $|x|$. & $|x|$. & $|x|$. & $P(x)$. & $P \times 1$. & $|x|$. & جلسه اول \\
\hline$P \times 1$ & $f \times l$. & $|x|$. & $f(x)$. & $f(x)$. & $P x \mid$. & $f \times 1$. & $|x|$. & جلسه دوم \\
\hline$P \times 1$ & $P \times 1$. & $|x|$. & $p \times 1$. & $f(x)$. & $|x|$. & $|x|$. & $|x|$. & جلسه سوم \\
\hline دوازدهيم & يازدهم & هم & فهم & هشتيم & هفتم & جهاروم & اول & هُته / شماره تمرين \\
\hline reir & $r \times I r$ & $1 \times 1$ & fxir & $P X \mid Y$ & fXIr & PXIr & Pxir & جلسه اول \\
\hline rxir & $r \times I r$ & $|x|$. & fxir & RXIY & fxir & fxir & fxir & جلسه دوم \\
\hline rxir & $r \times i r$ & $|x|$. & PXIr & PXIY & PXIr & PXIY & PXIr & جلسه سوم \\
\hline fxir & PXIY & $|x|$. & Pxir & PXIY & Pxir & PXIr & Fxir & جلسه اول \\
\hline PXIY & PXIY & $P \times 1$. & Pxir & PXIY & Pxir & PXIY & pxir & جلسه دوم \\
\hline PXIY & pxir & $|x|$. & pxir & PXIY & PXIY & pxir & reit & جلسه سوم \\
\hline
\end{tabular}

مجله بيومكانيك ورنش

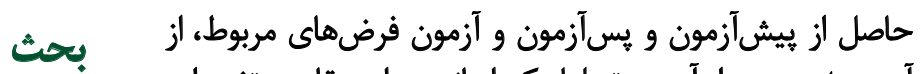

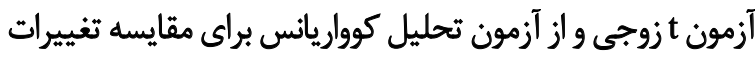

هدف تحقيق حاضر بررسى تأثير هشت هفته تمرينات اصلاحى ئي

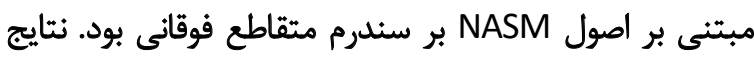

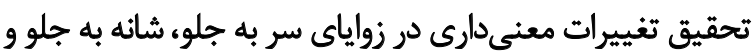

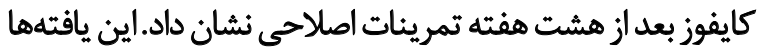

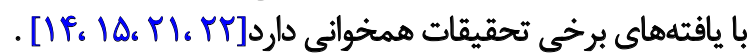
الكوى سندرم متقاطع فوقانى معمولاً در اقرادى كه در بر

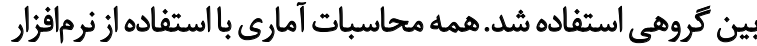
نسخه Y Y در سطح معنى دارى (1 SPSS بر اساس اطلاعات موجود در جدول شماره بّ برنامه تمرينات

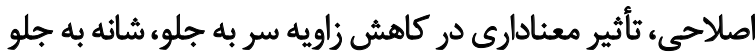

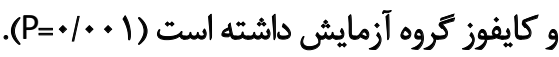


جدول ז. اطلاعات فردي شركت كنئدكان (انحراف استائداردثميانكين)

\begin{tabular}{|c|c|c|}
\hline ميانكَين +انحراف استاندارد & كروه & شاخص اندازهيرى \\
\hline $\begin{array}{l}r \cdot / \bullet \bullet \pm Y / . . \\
r \cdot / \Delta r \pm 1 / \Delta \Delta\end{array}$ & كتثرل & سن (سالل) \\
\hline 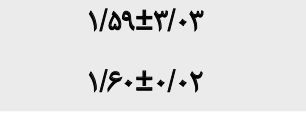 & كنثرل & قد (سانثىمتر) \\
\hline $\begin{array}{l}\Delta N \mid r \pm \Delta / \cdot \Lambda \\
\varepsilon \cdot|\Delta| \pm+/ \mid\end{array}$ & كنترل & 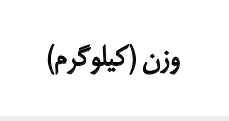 \\
\hline $\begin{array}{l}r / M I \pm 1 / 41 \\
M / Q I \pm 1 / T+\end{array}$ & كنترل & شاخص توده بدنى \\
\hline
\end{tabular}

مجله بيومكانيك وزنش

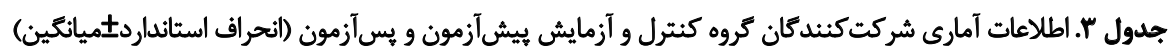

\begin{tabular}{|c|c|c|c|c|c|c|c|c|}
\hline \multicolumn{4}{|c|}{ آزمايش (0 انفر) } & \multicolumn{4}{|c|}{ كنترل (10 نفر) } & \multirow{3}{*}{ عروه } \\
\hline \multicolumn{8}{|c|}{ ميانئين +انحراف الستاندارد } & \\
\hline (Pilot Study) & $\mathbf{T}$ & يس آزمون & يشئزأمون & $\mathbf{P}$ & $\mathbf{T}$ & يسأزمون & بيش آزمون & \\
\hline$+1 \cdot+1^{*}$ & Ir/me & $p+/ p \pm 1 / p q$ & $\Delta .1+ \pm 1 / Y \wedge$ & . M & $-1 / M$ & $P q / P+r / * V$ & $p q \Delta \pm m /+1$ & سر به جلو \\
\hline $.1 \cdot 1^{*}$ & $r \cdot / \pi r$ & $\Delta \cdot / \Delta \pm \cdot / 9$ & $\Delta \Delta / + \pm 1 / \bullet \varnothing$ & $\cdot M$ & $-\cdot / / r$ & $\Delta \Delta / \backslash \pm I / \%$ & $\Delta \Delta / + \pm 1 / \Delta$ & ش مائه به جلو \\
\hline$+1++1^{*}$ & $r q / \mathrm{rA}$ & $H T / F E \pm 1 / F A$ & FNY $\pm 1 / F E$ & . $/ M$ & $-1 / \pi$ & $F V / I \pm T /+F$ & $\mid F / \cdot \pm r /+\varepsilon$ & كايفوز \\
\hline
\end{tabular}

مجله بيومكانيك ورنش

را علت تأثيركذارى تمرينات اصلاحى تحقيق خود بيان كردهاند

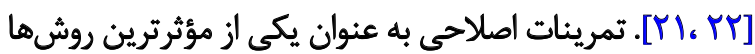

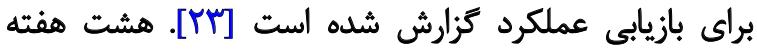

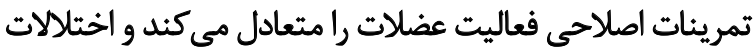

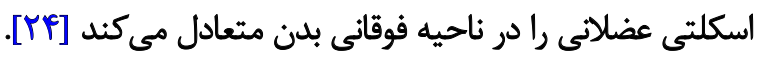

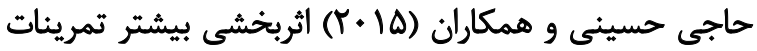

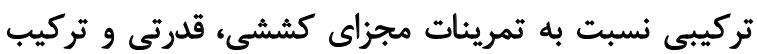

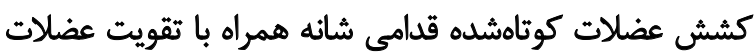

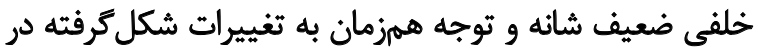

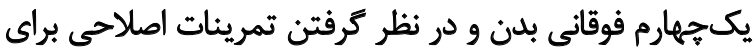

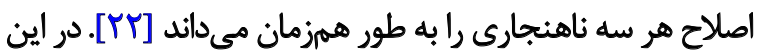

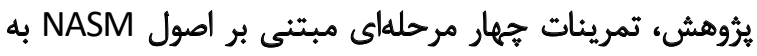

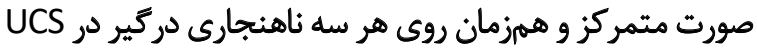

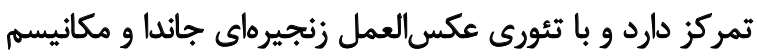

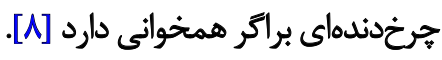

سرمن، كليات سندرمهاى اختلال حركتى بدن را منتشر كرد،

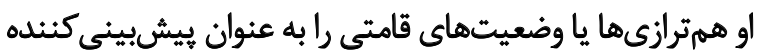

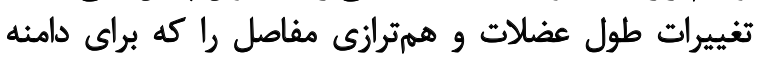

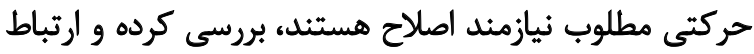

دورهاى زمانى طولانىمدت مي نشيند يا افرادى كه الكوهاى

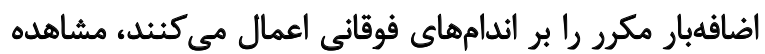

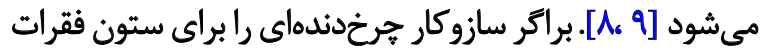
اينكونه توصيف كرد كه وضعيت بدنى نشسته ضعيف سبب

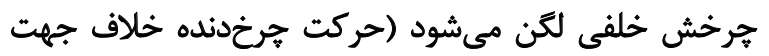

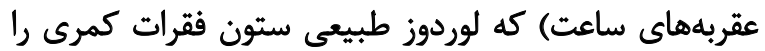

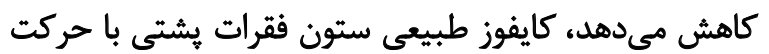

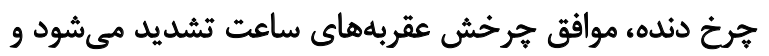

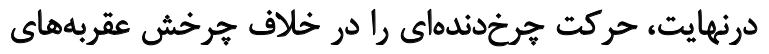

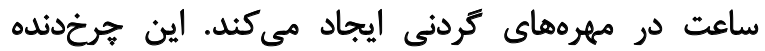
انتهايى است كه سبب ايجاد وضعيت قراركيرى رو به به جلوى إنى

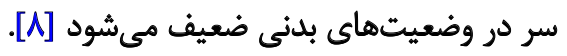

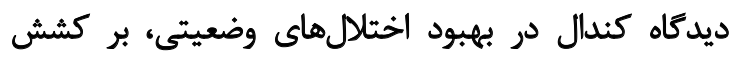

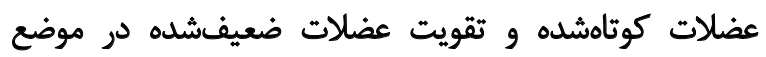

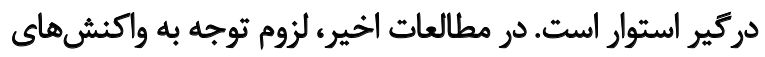

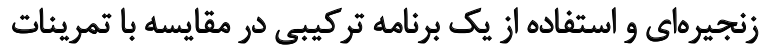

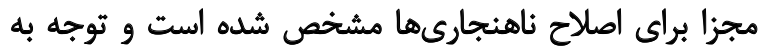
تغييرات همززمان شكل

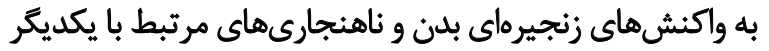


هدف كرفتند كه باعث بهبود يوسجر سر به جلو جلو شدهاند [1/]

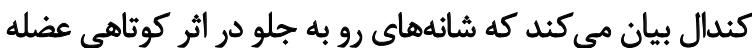

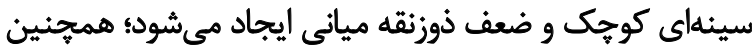

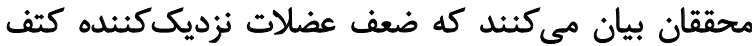

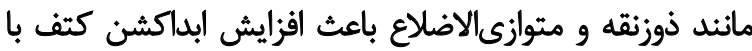

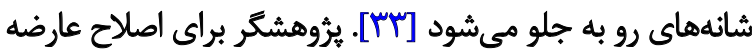

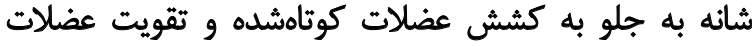

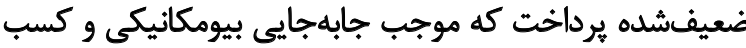

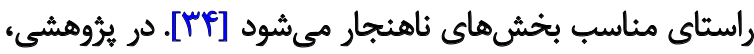

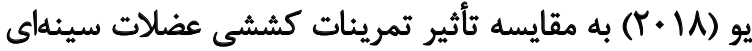

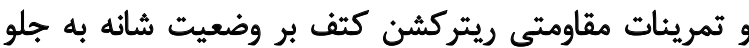

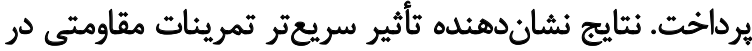

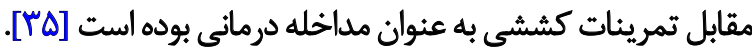

به نظر مىرسد بيىتمرينى موجب ضعيف شدن عضلات

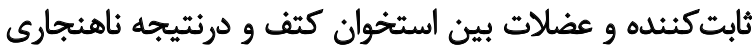

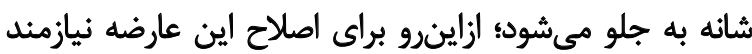

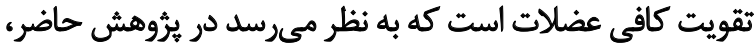

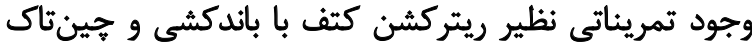

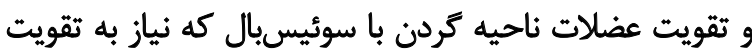

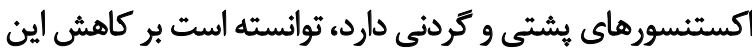

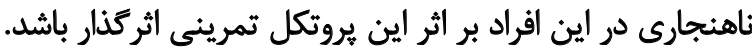

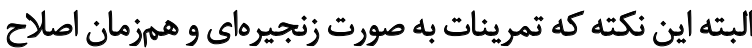

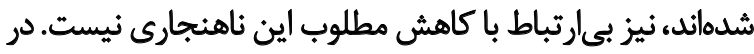

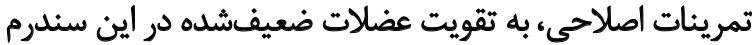

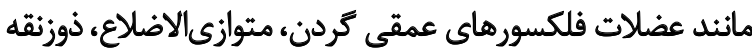

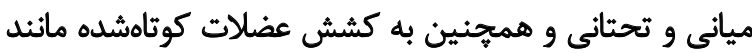

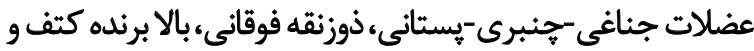

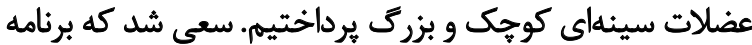

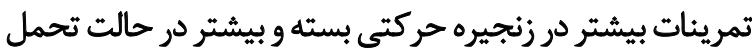

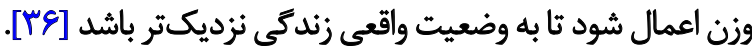

محققان بيان كردهاند كه تمرينات قدرتى، طول تاندون عضلات

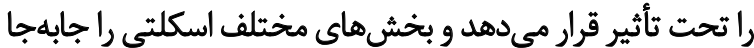

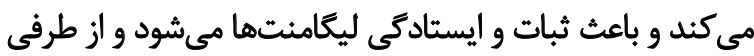

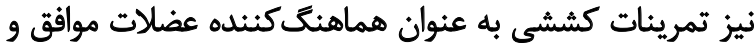

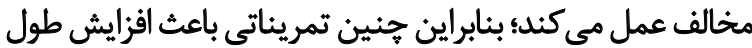

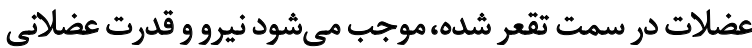

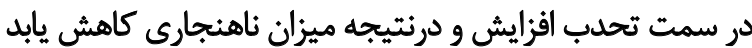

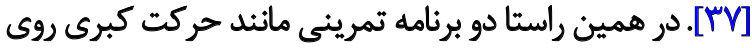

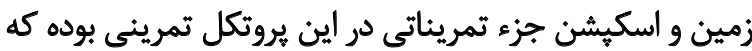

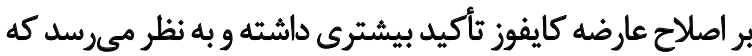

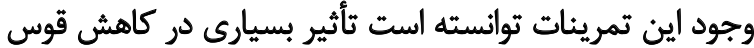

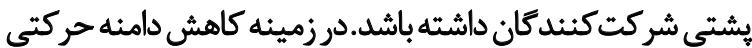

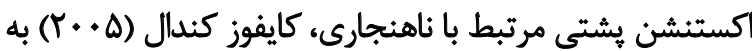
كاهش دامنه حركتى سينهاى شكمى مرتبط با كايفوز اشاره
معنادارى را بين كايفوز و سر به جلو و سندرم تحت آخرومي مكي

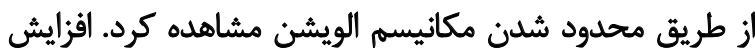

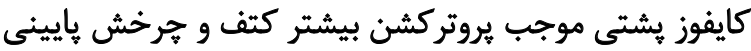

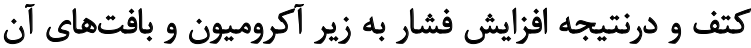

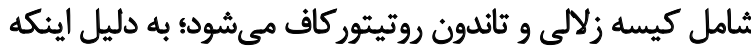

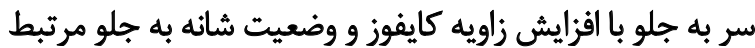

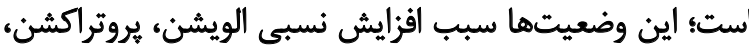

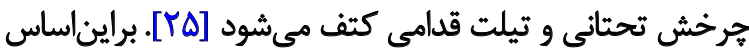

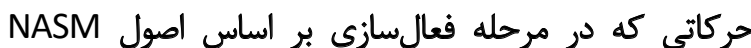

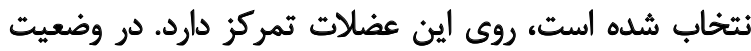

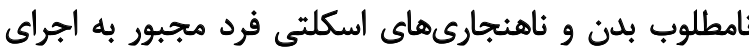

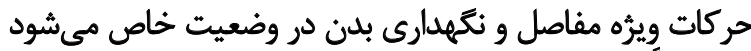

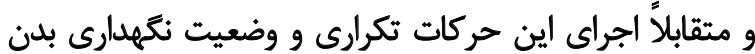

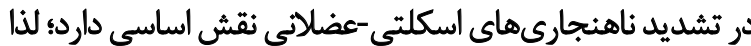

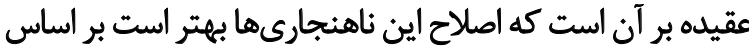

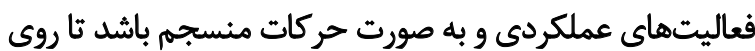

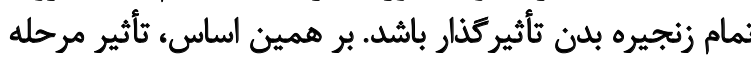

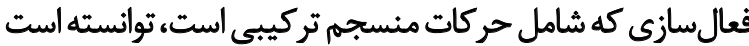
نقش مهمى در بهبود وضعيت ناهنجارى ايفا كند.

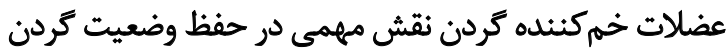

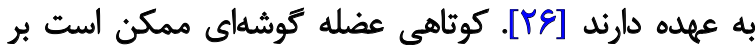

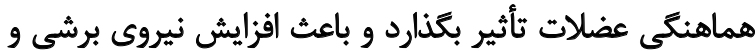

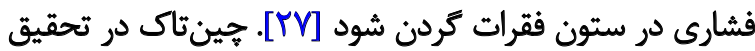

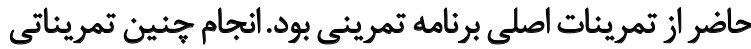

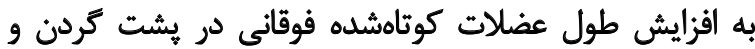

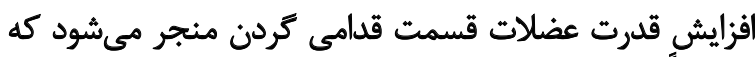

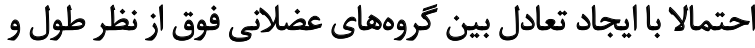

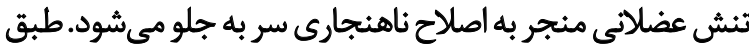

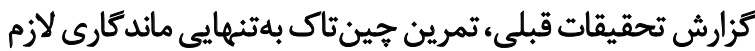

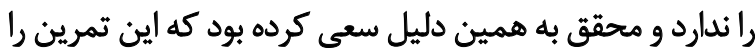

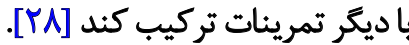

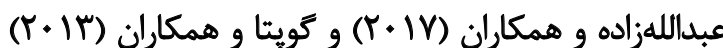

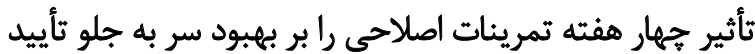

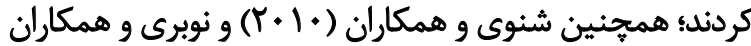
(T-1/N)

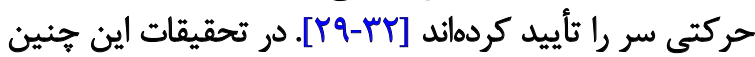

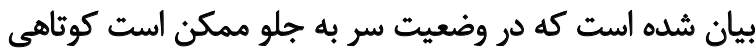

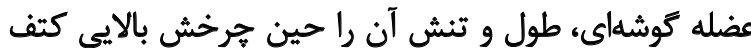

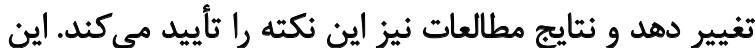

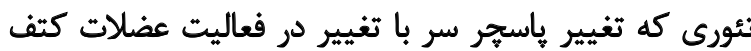

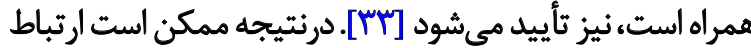

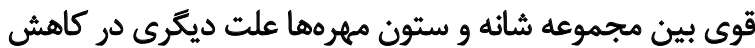

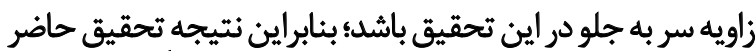

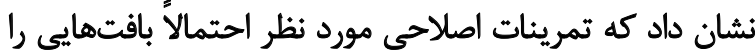




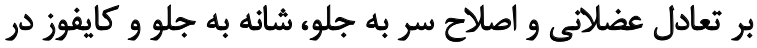

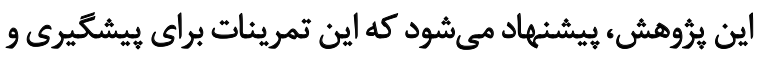
اصلاح UCS به طور منظم به كار كرفته شود.

\section{ملاحظات اخلاقي

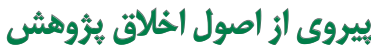

يثروهش حاضر بركرفته از باياننامه كارشناسىارشد رشته

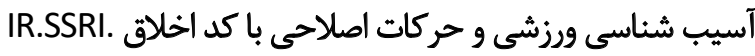
REC.1398.628 ازيروهشكاه تربيتبدنى و علوم ورزشى است.

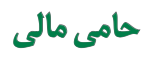

اين مقاله بركرفته از هاياننامه كارشناسىارشد نويسنده اول،

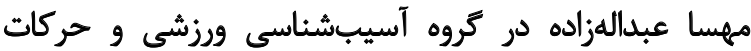
اصلاحى، مؤسسه آموزش عالى شفق تنكابن است.

$$
\text { مشاركت نويسند مَان }
$$

مفهومسازى، روششناسى، اعتبارسنجى، تحليل، تحقيق و

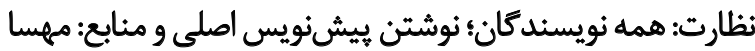
عبدالهزاده؛ مرور، بررسى و ويرايش: حسن نوشين دانشمندى.

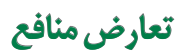

بنابر اظهار نويسندكان اين مقاله تعارض منافع ندارد.

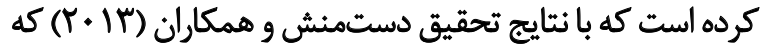

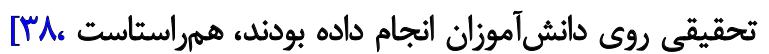

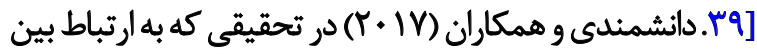

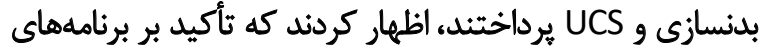

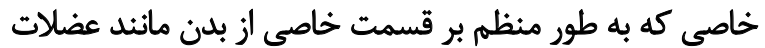

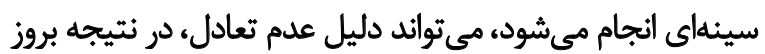

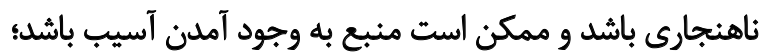

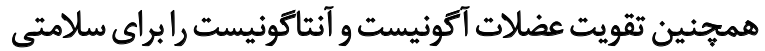

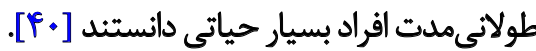
استفادهاز تكنيكهاى رهاسازى مايوفاشيال،به ايجاد يك پاسخ

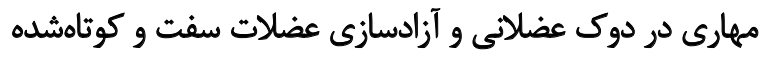

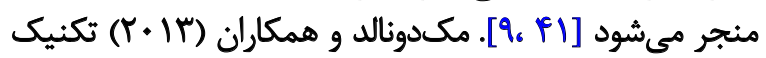

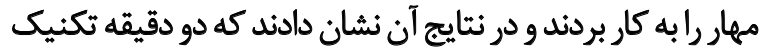

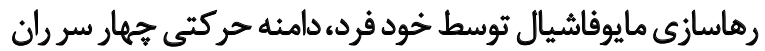

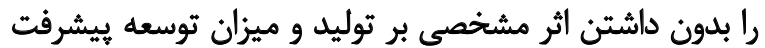

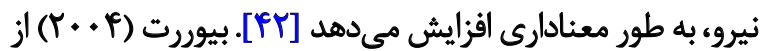

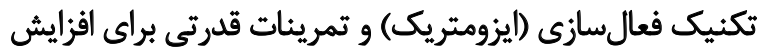

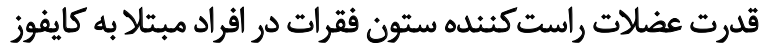

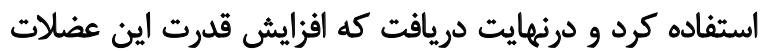

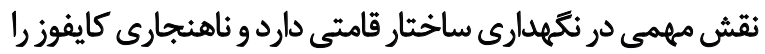

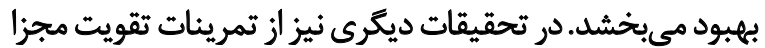

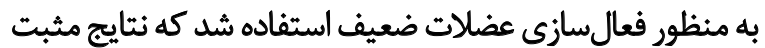

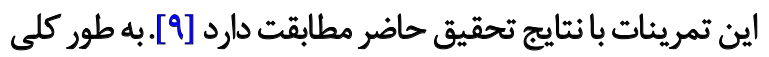

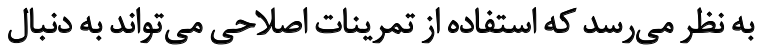

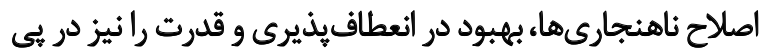

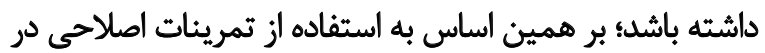

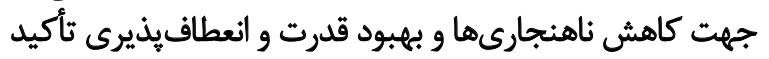

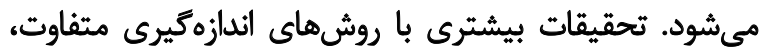

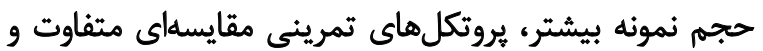

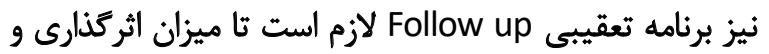
ماندكًارى اثر اين تمرينات بيشتر معلوم شود.

\section{تتيجهَيرى نهايى}

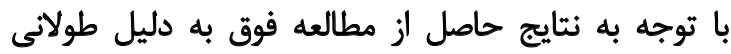

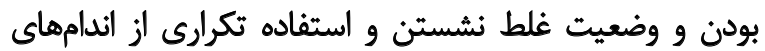

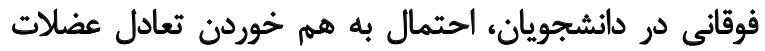

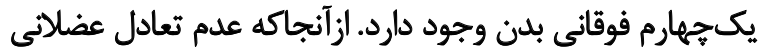

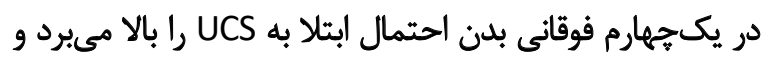

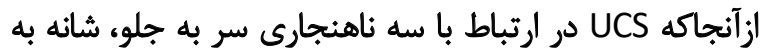

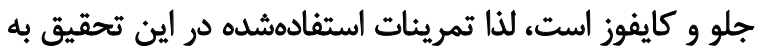

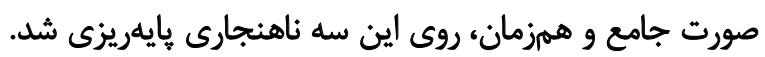

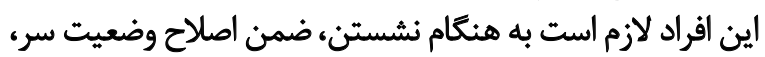

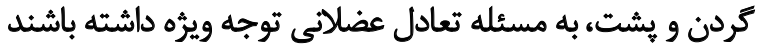

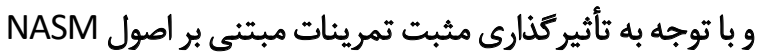




\section{References}

[1] Kargarfard M, Mahdavi-Nejad R, Ghasemi G-A, Rouzbehani R, Ghias M, Mahdavi-Jafari Z, et al. Assessment of Spinal Curvature in Isfahan University Students. J Isfahan Med Sch. 2010; 27(102):762-76.

[2] Balogh I, Ohlsson K, Nordander C, Björk J, Hansson G-Å. The importance of work organization on workload and musculoskeletal health-grocery store work as a model. Appl Ergon. 2016; 53:143-51. [DOI:10.1016/j. apergo.2015.09.004] [PMID]

[3] Salehi S, Hedayati R, Ghorbani R. The comparative study of the effect of stabilization exercise and stretching-strengthening exercise on balance parameters in forward head posture patients. J Rehabil. 2013; 14(1):5060.

[4] Moore MK. Upper crossed syndrome and its relationship to cervicogenic headache. J Manipulative Physiol Ther. 2004; 27(6):414-20. [DOI:10.1016/j.jmpt.2004.05.007] [PMID]

[5] Morris CE, Bonnefin D, Darville C. The Torsional Upper Crossed Syndrome: A multi-planar update to Janda's model, with a case series introduction of the mid-pectoral fascial lesion as an associated etiological factor. J Bodyw Mov Ther. 2015; 19(4):681-9. [DOI:10.1016/j. jbmt.2015.08.008] [PMID]

[6] Ghamkhar L, Kahlaee AH. Is forward head posture relevant to cervical muscles performance and neck pain? A case-control study. Braz J Phys Ther. 2019; 23(4):346-54. [DOI:10.1016/j.bjpt.2018.08.007] [PMID] [PMCID]

[7] Hasan NMA, Abdelrahman TEF. MRI evaluation of TMJ internal derangement: degree of anterior disc displacement correlated with other TMJ soft tissue and osseous abnormalities. Egypt J Radiol Nucl Med. 2014; 45(3):735-44. [DOI:10.1016/j.ejrnm.2014.03.013]

[8] Frank C, Page P, Lardner R. Assessment and treatment of muscle imbalance: The Janda approach. $1^{\text {st }}$ edition. Champaign, IL: Human kinetics; 2009.

[9] Clark M, Lucett S. NASM essentials of corrective exercise training: Philadelphia: Lippincott Williams \& Wilkins; 2010

[10] Magee DJ. Orthopedic physical assessment. E-Book. Amsterdam: Elsevier Health Sciences; 2014

[11] Lederman E. 14 - Neuromuscular rehabilitation: summary. Neuromuscul Rehabil Man Phys Ther . 2010; 169-71. [DOI:10.1016/B978-0-44306969-7.00014-0]

[12] Cole AK, McGrath ML, Harrington SE, Padua DA, Rucinski TJ, Prentice WE. Scapular bracing and alteration of posture and muscle activity in overhead athletes with poor posture. J Athl Train. 2013; 48(1):12-24. [DOI:10.4085/1062-6050-48.1.13] [PMID] [PMCID]

[13] El-Hamalawy FA. Forward head correction exercises for management of myogenic tempromandibular joint dysfunction. J Am Sci. 2011; 7(8):71-7.

[14] Roshani S, Mahdavinejad R, Ghanizadehesar N. The effect of a NASM-based training protocol on upper cross syndrome in paraplegia spinalcord injury patients. Sci J Ilam Univ Med Sci. 2018; 25(6):73-85. [DOI:10.29252/sjimu.25.6.73]

[15] Bae W-S, Lee H-O, Shin J-W, Lee K-C. The effect of middle and lower trapezius strength exercises and levator scapulae and upper trapezius stretching exercises in upper crossed syndrome. J Phys Ther Sci. 2016; 28(5):1636-9. [DOI:10.1589/jpts.28.1636] [PMID] [PMCID]

[16] Thigpen CA, Padua DA, Michener LA, Guskiewicz K, Giuliani C, Keener JD, et al. Head and shoulder posture affect scapular mechanics and mus- cle activity in overhead tasks. J Electromyogr Kinesiol. 2010; 20(4):701-9. [DOI:10.1016/j.jelekin.2009.12.003] [PMID]

[17] Seidi F, Rajabi R, Ebrahimi I, Alizadeh MH, Minoonejad H. The efficiency of corrective exercise interventions on thoracic hyper-kyphosis angle. J Back Musculoskelet Rehabil. 2014; 27(1):7-16. [DOI:10.3233/ BMR-130411] [PMID]

[18] Foad S. The effect of a 12-week corrective exercises program on forward head and shoulder deformities. Res Sport Med. 2013; 5(14):31-44.

[19] Silva AG, Johnson MI. Does forward head posture affect postural control in human healthy volunteers? Gait Posture. 2013; 38(2):352-3. [DOI:10.1016/j.gaitpost.2012.11.014] [PMID]

[20] Ebaugh DD, Spinelli BA. Scapulothoracic motion and muscle activity during the raising and lowering phases of an overhead reaching task. J Electromyogr Kinesiol. 2010; 20(2):199-205. [DOI:10.1016/j.jelekin.2009.04.001] [PMID]

[21] Daneshmandi H, Mogharabi Mozafari M. The effect of eight weeks of comprehensive corrective training on upper cross_syndrome. J Res Sport Med Technol. 2014; 12(7):75-86.

[22] Hajihosseini E, Norasteh A, Shamsi A, Daneshmandi H. The comparison of effect of three programs of strengthening stretching and comprehensive on upper crossed syndrome. J Rehabil Sci. 2015; 11(1):51-61.

[23] Armijo-Olivo S. A new paradigm shift in musculoskeletal rehabilitation: Why we should exercise the brain? Braz J Phys Ther. 2018; 22(2):95 [DOI:10.1016/j.bjpt.2017.12.001] [PMID] [PMCID]

[24] Arshadi R, Ghasemi GA, Samadi H. Effects of an 8-week selective corrective exercises program on electromyography activity of scapular and neck muscles in persons with upper crossed syndrome: Randomized controlled trial. Phys Ther Sport. 2019; 37:113-9. [DOI:10.1016/j. ptsp.2019.03.008] [PMID]

[25] Sahrmann S, Azevedo DC, Van Dillen L. Diagnosis and treatment of movement system impairment syndromes. Braz J Phys Ther. 2017 21(6):391-9.

[26] Kang DY. Deep cervical flexor training with a pressure biofeedback unit is an effective method for maintaining neck mobility and muscular endurance in college students with forward head posture. J Phys Ther Sci. 2015; 27(10):3207-10. [DOI:10.1589/jpts.27.3207] [PMID] [PMCID]

[27] Jeong H-J, Cynn H-S, Yi C-H, Yoon J-W, Lee J-H, Yoon T-L, et al. Stretching position can affect levator scapular muscle activity length, and cervical range of motion in people with a shortened levator scapulae. Phys Ther Sport. 2017; 26:13-9. [DOI:10.1016/j. ptsp.2017.04.001] [PMID]

[28] Beneka A, Malliou P, Gioftsidou A. Neck pain and office workers: An exercise program for the workplace. ACSM's Health \& Fitness J. 2014 18(3):18-24. [DOI:10.1249/FIT.0000000000000034]

[29] Gupta BD, Aggarwal S, Gupta B, Gupta M, Gupta N. Effect of deep cervical flexor training vs. conventional isometric training on forward head posture, pain, neck disability index in dentists suffering from chronic neck pain. J Clin Diagn Res (JCDR). 2013; 7(10):2261-4. [DOI:10.7860/ JCDR/2013/6072.3487] [PMID] [PMCID]

[30] Abdollahzade Z, Shadmehr A, Malmir K, Ghotbi N. Effects of 4 week postural corrective exercise on correcting forward head posture. J Mod Rehabil. 2017; 11(2):85-92.

[31] Shenoy S, Sodhi J, Sandhu JS. Effectiveness of strengthening exercises in the management of forward head posture among computer professionals. Indian J Physiother Occup Ther. 2010; 4(3):37-41. 
[32] Nobari M, Arslan SA, Hadian MR, Ganji B. Effect of corrective exercises on cervicogenic headache in office workers with forward head posture. J Mod Rehabil. 2017; 11(4):201-8.

[33] Weon J-H, Oh J-S, Cynn H-S, Kim Y-W, Kwon O-Y, Yi C-H. Influence of forward head posture on scapular upward rotators during isometric shoulder flexion. J Bodyw Mov Ther. 2010; 14(4):367-74. [DOI:10.1016/j. jbmt.2009.06.006] [PMID]

[34] Lynch SS, Thigpen CA, Mihalik JP, Prentice WE, Padua D. The effects of an exercise intervention on forward head and rounded shoulder postures in elite swimmers. Br J Sports Med. 2010; 44(5):376-81. [DOI:10.1136/bjsm.2009.066837] [PMID]

[35] Yoo W-g. Comparison of the effects of pectoralis muscles stretching exercise and scapular retraction strengthening exercise on forward shoulder. J Phys Ther Sci. 2018; 30(4):584-5. [DOI:10.1589/jpts.30.584] [PMID] [PMCID]

[36] Vaughn DW, Brown EW. The influence of an in-home based therapeutic exercise program on thoracic kyphosis angles. J Back Musculoskelet Rehabil. 2007; 20(4):155-65. [DOl:10.3233/BMR-2007-20404]

[37] Yoo W-g. Effect of thoracic stretching, thoracic extension exercise and exercises for cervical and scapular posture on thoracic kyphosis angle and upper thoracic pain. J Phys Ther Sci. 2013; 25(11):1509-10. [DOI:10.1589/jpts.25.1509] [PMID] [PMCID]

[38] Dastmanesh S, Eskandari E, Shafiee GH. Relationship between physical fitness abilities, trunk range of motion and kyphosis in junior high school students. Middle-East J Sci Res. 2013; 13(1):79-84.

[39] Peterson-Kendall F, Kendall-McCreary E, Geise-Provance P, McIntyreRodgers M, Romani W. Muscles: Testing and function, with posture and pain. US: Philadelphia: Lippincott Williams \& Wilkins, Ltd; 2005.

[40] Daneshmandi H, Harati J, Fahim Poor S. Bodybuilding links to upper crossed syndrome. Phys Act Rev. 2017; 5:124-31. [DOl:10.16926/ par.2017.05.17]

[41] Elhag E, Musa A, Sulieman A, Ahmed A. Nociceptive masseter inhibitory reflex in patients with myofascial tempromandibular disorders and healthy controls. J Neurol Sci. 2017; 381(Suppl):488. [DOI:10.1016/j. jns.2017.08.3583]

[42] MacDonald GZ, Penney MD, Mullaley ME, Cuconato AL, Drake CD, Behm DG, et al. An acute bout of self-myofascial release increases range of motion without a subsequent decrease in muscle activation or force. J Strength Cond Res. 2013; 27(3):812-21. [DOI:10.1519/ JSC.0b013e31825c2bc1] [PMID] 